\title{
Article \\ Body Mass and Emotional Eating: Emotional Eater Questionnaire (EEQ) in the Polish Adolescents' COVID-19 Experience (PLACE-19) Study
}

\author{
Dominika Skolmowska ${ }^{1}$ (D), Dominika Głąbska ${ }^{1, *(D)}$ and Dominika Guzek ${ }^{2}$ (D) \\ 1 Department of Dietetics, Institute of Human Nutrition Sciences, Warsaw University of Life \\ Sciences (SGGW-WULS), 159C Nowoursynowska Street, 02-776 Warsaw, Poland; \\ dominika_skolmowska@sggw.edu.pl \\ 2 Department of Food Market and Consumer Research, Institute of Human Nutrition Sciences, \\ Warsaw University of Life Sciences (SGGW-WULS), 159C Nowoursynowska Street, 02-776 Warsaw, Poland; \\ dominika_guzek@sggw.edu.pl \\ * Correspondence: dominika_glabska@sggw.edu.pl; Tel.: +48-22-593-71-26
}

\section{check for}

updates

Citation: Skolmowska, D.; Głąbska,

D.; Guzek, D. Body Mass and

Emotional Eating: Emotional Eater Questionnaire (EEQ) in the Polish Adolescents' COVID-19 Experience (PLACE-19) Study. Nutrients 2022, 14, 828. https://doi.org/10.3390/ nu14040828

Academic Editors: Livio Luzi, Gian Vincenzo Zuccotti, Ileana

Marina Terruzzi and Anna Ferrulli

Received: 13 January 2022

Accepted: 14 February 2022

Published: 16 February 2022

Publisher's Note: MDPI stays neutral with regard to jurisdictional claims in published maps and institutional affiliations.

Copyright: (C) 2022 by the authors. Licensee MDPI, Basel, Switzerland. This article is an open access article distributed under the terms and conditions of the Creative Commons Attribution (CC BY) license (https:// creativecommons.org/licenses/by/ $4.0 /)$.

\begin{abstract}
Stress caused by the Coronavirus Disease 2019 (COVID-19) pandemic may lead to emotional eating which may have a negative impact on the weight status. This study aimed to analyze the association between emotional eating and body mass, as well as changes in body mass during the COVID-19 pandemic, within the Polish Adolescents' COVID-19 Experience (PLACE-19) Study. A total of 1126 Polish adolescents, aged 15-20, were included. A random quota sampling was performed within a national sample, and emotional eating was assessed using Emotional Eater Questionnaire (EEQ). Based on the declared height and weight before and during the pandemic, the respondents were categorized according to their body mass (malnourished, normal weight, overweight, obese) and changes in body mass during the COVID-19 pandemic (lost weight, no body mass change, gained weight). Higher EEQ scores were achieved by female respondents compared with males $(p<0.0001)$, respondents who stated that they gained weight during the pandemic compared with those who stated either weight loss or no body mass change $(p<0.0001)$, and overweight and obese respondents compared with those who had normal weight and who were malnourished $(p<0.0001)$. A higher share of emotional eaters and very emotional eaters was found among female respondents, respondents stating weight gain during the pandemic, and overweight and obese respondents, compared with the other ones $(p<0.0001)$. Based on the findings, it may be concluded that among Polish adolescents gender, body mass, and body mass change during the COVID-19 pandemic are the major determinants of emotional eating behaviors and that female individuals, obese individuals, and those gaining weight are especially vulnerable to emotional eating behaviors. The results of the study suggest that the issue of emotional eating should be addressed in general public health policy and appropriate education should be provided to vulnerable groups such as female and obese adolescents.
\end{abstract}

Keywords: emotional eating; emotional eater; Emotional Eater Questionnaire (EEQ); body mass; body mass changes; national study; population-based study; adolescents; PLACE-19 Study

\section{Introduction}

Emotional eating is defined as the tendency to eat in response to negative emotions, while the food products chosen for eating are mostly energy-dense [1], and high in sugars and fat [2]. Emotional eating has been observed in both individuals with a normal weight and those who are overweight/obese [3], and may be considered a maladaptive coping strategy [4]. As such eating behavior involves the consumption of high-calorie food products, it may even have an impact on weight status, as indicated by in the review of Frayn \& Knäuper [5] that emotional eating in adults is associated with weight gain over 
time. However, a systematic review based on adolescents by Limbers \& Summers [6] did not confirm this relationship.

The outbreak of the Coronavirus Disease 2019 (COVID-19) pandemic has caused profound psychological distress worldwide [7]. It has been shown that stress may induce changes in eating habits and may lead to either an increase or a decrease in food intake, as it was proven in the case of children and adolescents by the systematic review and metaanalysis by Hill et al. [8]. Furthermore, psychological stress, resulting from the COVID-19 pandemic and associated with anxiety, fear, and isolation [9], has been found to increase the risk of obesity, due to its influence on not only eating behaviors but also on the immune and endocrine system [10].

Emotional eating is mainly assessed using dedicated questionnaires, including Emotional Eating Scale (EES) [11], Salzburg Emotional Eating Scale (SEES) [12], or Emotional Overeating Questionnaire (EOQ) [13]. However, one of the most commonly used psychological tools for the assessment of emotional eating is the Emotional Eater Questionnaire (EEQ) developed by Garaulet et al. [14]. EEQ has already been recognized as a reliable tool and it has been used by various researchers during the period of the COVID-19 pandemic $[15,16]$. For instance, Özcan \& Yeşillkaya [15], who conducted a study on Turkish adults, indicated that during the COVID-19 pandemic a higher share of participants adopted emotional eating behaviors, compared to the prepandemic period. In the study of López-Moreno et al. [16], carried out on Spanish adults, during the COVID-19 confinement, $21.8 \%$ and $11 \%$ of the study participants were claimed as emotional eaters or very emotional eaters, respectively.

As stated by the United Nations Children's Fund (UNICEF), the COVID-19 pandemic has brought along several problems, such as social isolation, uncertainty, fear, and increased screen time, all of which affect the mental health of children and adolescents [17]. It is also indicated that the pandemic may continue to have long-term adverse consequences for children and adolescents when compared to adults [18]. Additionally, although some studies have assessed emotional eating behaviors in adults during the period of COVID-19 pandemic $[15,16]$, to our best knowledge, the results of analogous studies on children and adolescents are scarce [19]. Moreover, as adolescence is a critical stage in terms of the development of emotional eating, it would be particularly important to investigate the influence of emotional eating on weight status among adolescents [20,21].

Taking the above into account, this study aimed to analyze the association between emotional eating and body mass, as well as changes in body mass during the COVID-19 pandemic, in a population-based sample of Polish adolescents, within the Polish Adolescents' COVID-19 Experience (PLACE-19) Study.

\section{Materials and Methods}

\subsection{Ethical Statement}

The study was conducted at the Institute of Human Nutrition Sciences, Warsaw University of Life Sciences (WULS-SGGW). The study was carried out in accordance with the guidelines laid down in the Declaration of Helsinki and all procedures involving human subjects received the approval of the Ethics Committee of the Central Clinical Hospital of the Ministry of Interior and Administration in Warsaw (No. 2/2021). Both participants and their parents or legal guardians provided written informed consent before participating in the study.

\subsection{Study Population}

The PLACE-19 Study, which analyzed the various aspects of the life of Polish adolescents (aged 15-20, a typical age for education in the secondary school) in the period of the COVID-19 pandemic, was divided into three phases. During the first phase of the study, the hygienic and personal protective behaviors of adolescents were investigated [22-24], while in the second and third phase their eating habits [25-30] and psychological aspects of eating behaviors were analyzed, respectively. 
The study of psychological aspects of eating behaviors was carried out from 21 January 2021 to 17 February 2021. Sampling was based on a random quota sampling method with quotas set for voivodeships and counties. The recruitment process included two stages: in the first stage, five counties were randomly selected (out of each 16 Polish voivodeships, resulting in a total of 80 counties), and in the second stage, five secondary schools were randomly selected (out of each randomly selected county, resulting in a total of 400 secondary schools).

The Headmaster of each selected secondary school was sent an invitation for the school to take part in the study. If the school was willing to participate, a link to the electronic version of the survey was sent.

The inclusion criteria for the study were as follows: female and male students of the randomly chosen secondary schools; aged 15-20 years; and informed consent obtained from both students and their parents/legal guardians.

The exclusion criteria were as follows: any missing or unreliable data in the questionnaire and participation in any previous phase of the PLACE-19 Study.

The procedure of sampling of secondary schools and students within the schools is described in detail in Figure 1. In the conducted study, a total number of 1126 adolescents participated, including 818 female and 308 male individuals. A higher proportion of girls than boys in nutritional studies is typical for Poland [31], and it was observed also in the other studies during the COVID-19 pandemic [32]. The mean age in the studied group was $16.7 \pm 1.13$ years (median of 17.0, differing from 15.0 to 20.0; nonparametric distribution), and it did not differ between female (16.7 \pm 1.14 years, median of 17.0, differing from 15.0 to 20.0; nonparametric distribution) and male individuals (16.7 \pm 1.10 years, median of 16.5, differing from 15.0 to 20.0; nonparametric distribution) (Mann-Whitney U test, $p=0.4110)$.

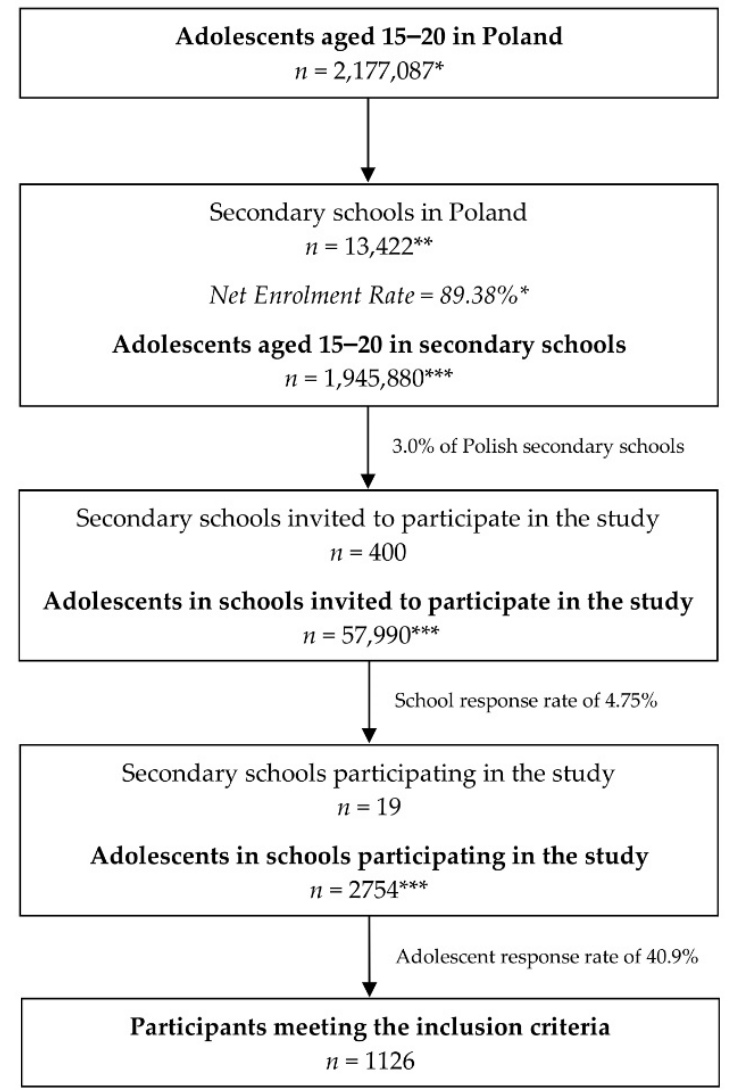

Figure 1. The detailed procedure of sampling of secondary schools and students within schools in the PLACE-19 Study; * data by the Central Statistical Office (CSO) in Poland [33,34]; ** data by the Polish Ministry of National Education [35]; ${ }^{* * *}$ calculated based on data by the CSO. 
In the online survey, each participant was asked about his/her body mass (in $\mathrm{kg}$ ) and height (in cm) for the period of COVID-19 pandemic (moment when the study was conducted, i.e., February 2021) as well as for the prepandemic period (March 2020 in Poland). For each respondent, for both periods the body mass index (BMI) values were calculated, using the Quetelet equation (body mass $(\mathrm{kg}) /$ height $^{2}\left(\mathrm{~m}^{2}\right)$ ) [36]. In the case of minor adolescents (aged <18 years), the BMI was analyzed based on the gender- and age-specific Polish growth reference values [37] using OLAF software (Children's Memorial Health Institute, Warsaw, Poland) [38] in order to specify the BMI percentile. In case of adolescents aged $>18$ years, the BMI values were directly analyzed. Based on the comparison of BMI percentiles (for adolescents aged $<18$ years) or BMI values (for adolescents aged $>18$ years) for the period of COVID-19 pandemic and for the prepandemic period, each adolescent was defined as gaining weight, loosing weight or maintaining stable weight during the COVID-19 pandemic.

Separately, each participant was categorized into the following groups: malnutrition, normal weight, overweight, and obesity. In the case of minor adolescents, the BMI was interpreted based on the World Health Organization (WHO, Geneva, Switzerland) growth reference cutoffs [39] set for children and adolescents, as follows: malnutrition-BMI $<5$ th percentile; normal weight-BMI $\Theta<5$ th-85th percentile); overweight-BMI $\Theta<85$ th-95th percentile); and obesity-BMI $\geq 95$ th percentile [40]. For other adolescents, the standard cutoffs fixed by WHO were applied [41].

\subsection{Applied Questionnaire}

When the PLACE-19 Study was conducted, education in all secondary schools was suspended and remote learning was introduced, as per the decision of the Polish Ministry of Education [42]. Therefore, all the data were collected using the computer-assisted web interview (CAWI) method. Students included in the study were provided with a link to the electronic version of the survey.

Emotional eating was assessed based on EEQ, developed and validated by Garaulet et al. [14]. EEQ is used to determine the extent to which eating behaviors are affected by emotions. The questionnaire consists of 10 questions:

- Do the weight scales have a great power over you? Can they change your mood?

- Do you crave specific foods?

- Is it difficult for you to stop eating sweet things, especially chocolate?

- Do you have problems controlling the amount of certain types of food you eat?

- Do you eat when you are stressed, angry or bored?

- Do you eat more of your favourite food and with less control when you are alone?

- Do you feel guilty when eat "forbidden" foods, like sweets or snacks?

- Do you feel less control over your diet when you are tired after work at night?

- When you overeat while on a diet, do you give up and start eating without control, particularly food that you think is fattening?

- How often do you feel that food controls you, rather than you controlling food?

Each question has four possible answers, namely: (1) never, (2) sometimes, (3) generally, and (4) always, which are scored from 1 to 3 points. The maximum points that can be obtained is 30, and the higher the points, the healthier are the eating behaviors. Based on the obtained points, four groups can be distinguished, according to the original version of the questionnaire: 0-5: non-emotional eater; 6-10: low emotional eater; 11-20: emotional eater; and 21-30: very emotional eater [14].

\subsection{Statistical Analysis}

The respondents were stratified based on their emotional eating, as well as based on the other determinants, in the following sub-groups:

- emotional eating-non-emotional eaters $(n=303)$, low emotional eaters $(n=386)$, emotional eaters $(n=377)$, very emotional eaters $(n=60)$;

- gender-female respondents $(n=818)$ and male respondents $(n=308)$; 
- declared body mass change during the COVID-19 pandemic - gained weight $(n=311)$, lost weight $(n=296)$ and no body mass change $(n=519)$;

- body mass - malnutrition $(n=39)$, normal weight $(n=842)$, overweight $(n=158)$, obesity $(n=87)$.

The normality of distribution was determined using Kolmogorov-Smirnov test. The comparison of the subgroups was performed using Mann-Whitney U test/ Kruskal-Wallis test (due to non-parametric distributions) and $\mathrm{chi}^{2}$ test.

The additional analysis for the detailed questions from EEQ was conducted while using multinomial logistic regression.

The statistical significance was set for the level of $p \leq 0.05$. The statistical analysis was done using Statistica version 13.3 (StatSoft Inc., Tulsa, OK, USA), Statgraphics Plus for Windows 5.1 (Statgraphics Technologies Inc., The Plains, VA, USA) and Jamovi 2.2.5 (https://www.jamovi.org/; accessed on 6 February 2022).

\section{Results}

The emotional eating scores calculated based on the EEQ and resultant categories in the PLACE-19 Study sample, depending on gender, on declared body mass change during the COVID-19 pandemic and on body mass are presented in Table 1. Female respondents, compared with male ones, were characterized by higher EEQ scores $(p<0.0001)$, indicating that they are more vulnerable to emotional eating behaviors than male ones. At the same time, respondents who stated gain weight during pandemic, compared with those who stated either weight loss or no body mass change, received higher EEQ scores $(p<0.0001)$ and obese respondents, compared with the other ones, received higher EEQ scores $(p<0.0001)$, indicating that a higher body mass and increase of body mass are commonly associated with emotional eating behaviors.

Table 1. The emotional eating scores calculated based on the Emotional Eater Questionnaire (EEQ) and resultant categories in the PLACE-19 Study sample $(n=1126)$, depending on gender, on declared body mass change during the COVID-19 pandemic and on body mass assessed based on the Body Mass Index (BMI).

\begin{tabular}{|c|c|c|c|c|c|c|c|c|}
\hline Characteristic & Mean \pm SD & $\begin{array}{c}\text { Median } \\
\text { (25th-75th) }\end{array}$ & $p^{*}$ & $\begin{array}{l}\text { Non-Emotional } \\
\text { Eaters }(n=303)\end{array}$ & $\begin{array}{l}\text { Low Emotional } \\
\text { Eaters }(n=386)\end{array}$ & $\begin{array}{c}\text { Emotional } \\
\text { Eaters }(n=377)\end{array}$ & $\begin{array}{c}\text { Very } \\
\text { Emotional } \\
\text { Eaters }(n=60)\end{array}$ & $p^{* *}$ \\
\hline \multicolumn{9}{|c|}{ Depending on gender } \\
\hline Female & $10.5 \pm 6.1$ & $10.0 *(6.0-14.0)$ & \multirow{2}{*}{$<0.0001$} & $182(22.2 \%)$ & $276(33.7 \%)$ & $305(37.3 \%)$ & $55(6.7 \%)$ & \multirow{2}{*}{$<0.0001$} \\
\hline Male & $7.6 \pm 5.3$ & $7.0 *(3.3-10.8)$ & & $121(39.3 \%)$ & $110(35.7 \%)$ & $72(23.4 \%)$ & $5(1.6 \%)$ & \\
\hline \multicolumn{9}{|c|}{ Depending on declared body mass change during the COVID-19 pandemic } \\
\hline Lost weight & $9.2 \pm 5.8$ & $9.0 *(5.0-13.0)^{b}$ & \multirow{3}{*}{$<0.0001$} & $171(32.9 \%)$ & $200(38.5 \%)$ & $130(25.0 \%)$ & $18(3.5 \%)$ & \multirow{3}{*}{$<0.0001$} \\
\hline $\begin{array}{l}\text { No body mass } \\
\text { change }\end{array}$ & $8.4 \pm 5.4$ & $8.0 *(5.0-11.0)^{\mathrm{b}}$ & & $77(26.0 \%)$ & $109(36.8 \%)$ & $98(33.1 \%)$ & $12(4.1 \%)$ & \\
\hline Gained weight & $12.1 \pm 6.4$ & $12.0 *(7.0-17.0)^{\mathrm{a}}$ & & $55(17.7 \%)$ & $77(24.8 \%)$ & $149(47.9 \%)$ & $30(9.6 \%)$ & \\
\hline \multicolumn{9}{|c|}{ Depending on body mass assessed based on the BMI } \\
\hline Malnourished & $6.7 \pm 4.3$ & $6.0(4.0-10.0)^{\mathrm{a}}$ & & $15(38.5 \%)$ & $16(41 \%)$ & $8(20.5 \%)$ & $0(0.0 \%)$ & \multirow{4}{*}{$<0.0001$} \\
\hline $\begin{array}{l}\text { Normal } \\
\text { weight }\end{array}$ & $9.3 \pm 5.9$ & $8.0 *(5.0-13.0)^{\mathrm{b}}$ & $<0.0001$ & $237(28.1 \%)$ & $300(35.6 \%)$ & $266(31.6 \%)$ & $39(4.6 \%)$ & \\
\hline Overweight & $10.5 \pm 6.5$ & $10.0 *(5.0-14.0)^{\mathrm{b}}$ & & $40(25.3 \%)$ & $49(31.0 \%)$ & $54(34.2 \%)$ & $15(9.5 \%)$ & \\
\hline Obese & $12.9 \pm 5.9$ & $12.0(9.0-18.0)^{\mathrm{c}}$ & & $11(12.6 \%)$ & $21(24.1 \%)$ & $49(56.3 \%)$ & $6(6.9 \%)$ & \\
\hline
\end{tabular}

* Mann-Whitney U test/ Kruskal-Wallis test; values with different letters $\left({ }^{a}, b, c\right)$ are significantly different; ** chi $^{2}$ test.

Within the sub-groups stratified based on gender, higher share of female respondents was classified as emotional eaters and very emotional eaters, while in male sub-group as non-emotional eaters and low emotional eaters $(p<0.0001)$. Within the sub-groups stratified based on body mass change, higher share of respondents who gained weight was classified as emotional eaters and very emotional eaters, while higher share of respondents who lost weight or observed no body mass change were classified as non-emotional eaters and low emotional eaters $(p<0.0001)$. Within the sub-groups stratified based on body mass, higher 
share of overweight and obese respondents, compared with the other ones, were classified as emotional eaters and very emotional eaters, while higher share of malnourished and normal weight respondents were classified as non-emotional eaters and low emotional eaters $(p<0.0001)$.

As all the studied factors were associated with EEQ, additionally, to verify if there is an association between the studied factors, the association between gender, the declared body mass change during the COVID-19 pandemic, and body mass were studied (chi ${ }^{2}$ test). The results of the analysis revealed that although gender was not associated with body mass $(p=0.4121)$, it was associated with body mass change $(p=0.0015)$. This indicates that gender-dependent differences and body mass change-dependent differences may be associated with each other. Similarly, the body mass was associated with body mass change $(p=0.0003)$, so the related differences of EEQ may be also associated with each other.

Taking into account observed results and the risk of interfering factors, for the detailed questions within EEQ, the estimated effects of selected predictors were assessed while using multinomial logistic regression.

The estimated effects of selected predictors using multinomial logistic regression for the declared frequency of confirmation for EEQ question 1 'Weight scales having a great power over respondents and changing their mood' in the PLACE-19 Study sample are presented in Table 2. The results of multinomial logistic regression indicated that declared frequency of confirmation of weight scales always having a great power over respondents and changing their mood was higher in female while compared with male respondents (OR 6.4050 (4.0081-10.2360); $p<0.001$ ), in respondents gaining weight while compared with those reporting no body mass change (OR $2.9530(1.8762-4.6460) ; p<0.001)$, and in obese respondents while compared with normal weight ones (OR 4.8740 (2.0897-11.3690); $p<0.001)$.

Table 2. Estimated effects of selected predictors using multinomial logistic regression for the declared frequency of confirmation for Emotional Eater Questionnaire (EEQ) question 1 'Weight scales having a great power over respondents and changing their mood' in the PLACE-19 Study sample $(n=1126)$.

\begin{tabular}{|c|c|c|c|c|c|c|c|c|c|c|}
\hline \multirow{2}{*}{ Variable } & \multirow{2}{*}{ Categories } & \multirow{2}{*}{$\begin{array}{c}\text { Declared } \\
\text { 'Sometimes' } \\
\text { OR } \\
(p \text {-Value) }\end{array}$} & \multicolumn{2}{|c|}{ 95\% Confidence Interval } & \multirow{2}{*}{$\begin{array}{c}\text { Declared } \\
\text { 'Generally' } \\
\text { OR } \\
(p-\text { Value })\end{array}$} & \multicolumn{2}{|c|}{$\begin{array}{l}\text { 95\% Confidence } \\
\text { Interval }\end{array}$} & \multirow{2}{*}{$\begin{array}{c}\text { Declared } \\
\text { 'Always' OR } \\
(p \text {-Value })\end{array}$} & \multicolumn{2}{|c|}{$\begin{array}{l}\text { 95\% Confidence } \\
\text { Interval }\end{array}$} \\
\hline & & & $\begin{array}{l}\text { Lower } \\
\text { Bound }\end{array}$ & $\begin{array}{l}\text { Upper } \\
\text { Bound }\end{array}$ & & $\begin{array}{l}\text { Lower } \\
\text { Bound }\end{array}$ & $\begin{array}{l}\text { Upper } \\
\text { Bound }\end{array}$ & & $\begin{array}{l}\text { Lower } \\
\text { Bound }\end{array}$ & $\begin{array}{l}\text { Upper } \\
\text { Bound }\end{array}$ \\
\hline \multirow[b]{2}{*}{ Gender } & Male (ref.) & - & - & - & - & - & - & - & - & - \\
\hline & Female & $\begin{array}{c}2.2400 \\
(<0.001)\end{array}$ & 1.6109 & 3.1160 & $\begin{array}{c}4.6380 \\
(<0.001)\end{array}$ & 3.0521 & 7.0460 & $\begin{array}{l}6.4050 \\
(<0.001)\end{array}$ & 4.0081 & 10.2360 \\
\hline \multirow{3}{*}{$\begin{array}{l}\text { Body mass } \\
\text { change }\end{array}$} & $\begin{array}{l}\text { No body } \\
\text { mass change } \\
\text { (ref.) }\end{array}$ & - & - & - & - & - & - & - & - & - \\
\hline & Lost weight & $1.4730(0.056)$ & 0.9907 & 2.1890 & $1.8770(0.006)$ & 1.1999 & 2.9360 & $\begin{array}{c}2.4480 \\
(<0.001)\end{array}$ & 1.5366 & 3.8990 \\
\hline & $\begin{array}{l}\text { Gained } \\
\text { weight }\end{array}$ & $1.1470(0.501)$ & 0.7689 & 1.7120 & $\begin{array}{c}2.2070 \\
(<0.001)\end{array}$ & 1.4280 & 3.4110 & $\begin{array}{c}2.9530 \\
(<0.001)\end{array}$ & 1.8762 & 4.6460 \\
\hline \multirow{4}{*}{ Body mass } & $\begin{array}{c}\text { Normal } \\
\text { weight (ref.) }\end{array}$ & - & - & - & - & - & - & - & - & - \\
\hline & Malnourished & $0.6550(0.321)$ & 0.2837 & 1.5110 & $0.8840(0.789)$ & 0.3568 & 2.1890 & $0.7050(0.506)$ & 0.2512 & 1.9770 \\
\hline & Overweight & $1.3030(0.270)$ & 0.8137 & 2.0880 & $1.2330(0.447)$ & 0.7180 & 2.1190 & $1.7040(0.051)$ & 0.9982 & 2.9100 \\
\hline & Obese & $2.5960(0.024)$ & 1.1355 & 5.9330 & $3.9700(0.001)$ & 1.7088 & 9.2250 & $\begin{array}{c}4.8740 \\
(<0.001)\end{array}$ & 2.0897 & 11.3690 \\
\hline
\end{tabular}

OR-Odds Ratio.

The estimated effects of selected predictors using multinomial logistic regression for the declared frequency of confirmation for EEQ question 2 'Craving specific foods' in the PLACE-19 Study sample are presented in Table 3. The results of multinomial logistic regression indicated that declared frequency of confirmation of always craving specific foods was higher in female while compared with male respondents (OR 2.3750 $(1.2917-4.3680) ; p=0.005)$. 
Table 3. Estimated effects of selected predictors using multinomial logistic regression for the declared frequency of confirmation for Emotional Eater Questionnaire (EEQ) question 2 'Craving specific foods' in the PLACE-19 Study sample $(n=1126)$.

\begin{tabular}{|c|c|c|c|c|c|c|c|c|c|c|}
\hline \multirow{2}{*}{ Variable } & \multirow{2}{*}{ Categories } & \multirow{2}{*}{$\begin{array}{c}\text { Declared } \\
\text { 'Sometimes' } \\
\text { OR } \\
(p \text {-Value })\end{array}$} & \multicolumn{2}{|c|}{$95 \%$ Confidence Interval } & \multirow{2}{*}{$\begin{array}{c}\text { Declared } \\
\text { ‘Generally' } \\
\text { OR } \\
\text { ( } p \text {-Value) }\end{array}$} & \multicolumn{2}{|c|}{$\begin{array}{c}\text { 95\% Confidence } \\
\text { Interval }\end{array}$} & \multirow{2}{*}{$\begin{array}{c}\text { Declared } \\
\text { 'Always' OR } \\
(p \text {-Value })\end{array}$} & \multicolumn{2}{|c|}{$\begin{array}{l}\text { 95\% Confidence } \\
\text { Interval }\end{array}$} \\
\hline & & & $\begin{array}{l}\text { Lower } \\
\text { Bound }\end{array}$ & $\begin{array}{l}\text { Upper } \\
\text { Bound }\end{array}$ & & $\begin{array}{l}\text { Lower } \\
\text { Bound }\end{array}$ & $\begin{array}{l}\text { Upper } \\
\text { Bound }\end{array}$ & & $\begin{array}{l}\text { Lower } \\
\text { Bound }\end{array}$ & $\begin{array}{l}\text { Upper } \\
\text { Bound }\end{array}$ \\
\hline \multirow{2}{*}{ Gender } & Male (ref.) & - & - & - & - & - & - & - & - & - \\
\hline & Female & $1.7890(0.017)$ & 1.1088 & 2.8850 & $2.1780(0.002)$ & 1.3349 & 3.5550 & $2.3750(0.005)$ & 1.2917 & 4.3680 \\
\hline \multirow{3}{*}{$\begin{array}{l}\text { Body mass } \\
\text { change }\end{array}$} & $\begin{array}{l}\text { No body } \\
\text { mass change } \\
\text { (ref.) }\end{array}$ & - & - & - & - & - & - & - & - & - \\
\hline & Lost weight & $0.4930(0.008)$ & 0.2912 & 0.8330 & $0.5830(0.048)$ & 0.3414 & 0.9960 & $0.7530(0.395)$ & 0.3918 & 1.4480 \\
\hline & $\begin{array}{l}\text { Gained } \\
\text { weight }\end{array}$ & $0.9370(0.834)$ & 0.5082 & 1.7270 & $1.3870(0.297)$ & 0.7499 & 2.5660 & $1.727(0.133)$ & 0.8472 & 3.5200 \\
\hline \multirow{4}{*}{ Body mass } & $\begin{array}{c}\text { Normal } \\
\text { weight (ref.) }\end{array}$ & - & - & - & - & - & - & - & - & - \\
\hline & Malnourished & $0.5330(0.205)$ & 0.2017 & 1.4100 & $0.4600(0.130)$ & 0.1683 & 1.2560 & $0.1200(0.053)$ & 0.0141 & 1.0310 \\
\hline & Overweight & $1.0140(0.965)$ & 0.5370 & 1.9160 & $0.6600(0.219)$ & 0.3407 & 1.2800 & $0.9320(0.858)$ & 0.4315 & 2.0130 \\
\hline & Obese & $1.4470(0.459)$ & 0.5443 & 3.8470 & $1.1090(0.839)$ & 0.4102 & 2.9960 & $1.3630(0.587)$ & 0.4457 & 4.1680 \\
\hline
\end{tabular}

OR-Odds Ratio.

The estimated effects of selected predictors using multinomial logistic regression for the declared frequency of confirmation for EEQ question 3 'Difficulty stopping eating sweet things, especially chocolate' in the PLACE-19 Study sample are presented in Table 4. The results of multinomial logistic regression indicated that declared frequency of confirmation of always having a difficulty stopping eating sweet things, especially chocolate, was higher in female while compared with male respondents (OR $2.0720(1.2318-3.4870)$; $p=0.006$ ), and in respondents gaining weight while compared with those reporting no body mass change (OR 2.6020 (1.5689-4.3170); $p<0.001)$.

Table 4. Estimated effects of selected predictors using multinomial logistic regression for the declared frequency of confirmation for Emotional Eater Questionnaire (EEQ) question 3 `Difficulty stopping eating sweet things, especially chocolate' in the PLACE-19 Study sample $(n=1126)$.

\begin{tabular}{|c|c|c|c|c|c|c|c|c|c|c|}
\hline \multirow{2}{*}{ Variable } & \multirow{2}{*}{ Categories } & \multirow{2}{*}{$\begin{array}{c}\text { Declared } \\
\text { 'Sometimes' } \\
\text { OR } \\
(p \text {-Value })\end{array}$} & \multicolumn{2}{|c|}{ 95\% Confidence Interval } & \multirow{2}{*}{$\begin{array}{c}\text { Declared } \\
\text { 'Generally' } \\
\text { OR } \\
(p \text {-Value) }\end{array}$} & \multicolumn{2}{|c|}{$\begin{array}{l}95 \% \text { Confidence } \\
\text { Interval }\end{array}$} & \multirow{2}{*}{$\begin{array}{c}\text { Declared } \\
\text { ‘Always' OR } \\
(p \text {-Value })\end{array}$} & \multicolumn{2}{|c|}{$\begin{array}{l}\text { 95\% Confidence } \\
\text { Interval }\end{array}$} \\
\hline & & & $\begin{array}{l}\text { Lower } \\
\text { Bound }\end{array}$ & $\begin{array}{l}\text { Upper } \\
\text { Bound }\end{array}$ & & $\begin{array}{l}\text { Lower } \\
\text { Bound }\end{array}$ & $\begin{array}{l}\text { Upper } \\
\text { Bound }\end{array}$ & & $\begin{array}{l}\text { Lower } \\
\text { Bound }\end{array}$ & $\begin{array}{l}\text { Upper } \\
\text { Bound }\end{array}$ \\
\hline \multirow{2}{*}{ Gender } & Male (ref.) & - & - & - & - & - & - & - & - & - \\
\hline & Female & $1.4870(0.012)$ & 1.0914 & 2.0250 & $1.4510(0.056)$ & 0.9899 & 2.1280 & $2.0720(0.006)$ & 1.2318 & 3.4870 \\
\hline \multirow{3}{*}{$\begin{array}{l}\text { Body mass } \\
\text { change }\end{array}$} & $\begin{array}{l}\text { No body } \\
\text { mass change } \\
\text { (ref.) }\end{array}$ & - & - & - & - & - & - & - & - & - \\
\hline & Lost weight & $0.8310(0.278)$ & 0.5950 & 1.1610 & $0.9970(0.988)$ & 0.6525 & 1.5220 & $\begin{array}{c}1.1290 \\
(0.6670)\end{array}$ & 0.6489 & 1.9650 \\
\hline & $\begin{array}{l}\text { Gained } \\
\text { weight }\end{array}$ & $1.0910(0.627)$ & 0.7683 & 1.5480 & $1.8150(0.004)$ & 1.2050 & 2.7350 & $\begin{array}{c}2.6020 \\
(<0.001)\end{array}$ & 1.5689 & 4.3170 \\
\hline \multirow{4}{*}{ Body mass } & $\begin{array}{c}\text { Normal } \\
\text { weight (ref.) }\end{array}$ & - & - & - & - & - & - & - & - & - \\
\hline & Malnourished & $0.7210(0.353)$ & 0.3610 & 1.4390 & $0.3160(0.067)$ & 0.0922 & 1.0850 & $0.2160(0.139)$ & 0.0283 & 1.6430 \\
\hline & Overweight & $0.9960(0.986)$ & 0.6543 & 1.5170 & $1.3000(0.285)$ & 0.8035 & 2.1030 & $1.5560(0.133)$ & 0.8738 & 2.7700 \\
\hline & Obese & $1.3580(0.285)$ & 0.7749 & 2.3780 & $1.3990(0.316)$ & 0.7262 & 2.6930 & $1.8620(0.099)$ & 0.8890 & 3.8990 \\
\hline
\end{tabular}

OR-Odds Ratio.

The estimated effects of selected predictors using multinomial logistic regression for the declared frequency of confirmation for EEQ question 4 'Problems controlling the amount of certain types of food they eat' in the PLACE-19 Study sample are presented in Table 5. The results of multinomial logistic regression indicated that declared frequency of 
confirmation of always having problems controlling the amount of certain types of food they eat was higher in respondents gaining weight while compared with those reporting no body mass change (OR 3.3635 (1.7222-6.5690); $p<0.001)$.

Table 5. Estimated effects of selected predictors using multinomial logistic regression for the declared frequency of confirmation for Emotional Eater Questionnaire (EEQ) question 4 'Problems controlling the amount of certain types of food they eat' in the PLACE-19 Study sample $(n=1126)$.

\begin{tabular}{|c|c|c|c|c|c|c|c|c|c|c|}
\hline \multirow{2}{*}{ Variable } & \multirow{2}{*}{ Categories } & \multirow{2}{*}{$\begin{array}{c}\text { Declared } \\
\text { 'Sometimes' } \\
\text { OR } \\
(p \text {-Value) }\end{array}$} & \multicolumn{2}{|c|}{ 95\% Confidence Interval } & \multirow{2}{*}{$\begin{array}{c}\text { Declared } \\
\text { 'Generally' } \\
\text { OR } \\
(p \text {-Value })\end{array}$} & \multicolumn{2}{|c|}{$\begin{array}{l}\text { 95\% Confidence } \\
\text { Interval }\end{array}$} & \multirow{2}{*}{$\begin{array}{c}\text { Declared } \\
\text { 'Always' OR } \\
\text { ( } p \text {-Value) }\end{array}$} & \multicolumn{2}{|c|}{$\begin{array}{l}\text { 95\% Confidence } \\
\text { Interval }\end{array}$} \\
\hline & & & $\begin{array}{l}\text { Lower } \\
\text { Bound }\end{array}$ & $\begin{array}{l}\text { Upper } \\
\text { Bound }\end{array}$ & & $\begin{array}{l}\text { Lower } \\
\text { Bound }\end{array}$ & $\begin{array}{l}\text { Upper } \\
\text { Bound }\end{array}$ & & $\begin{array}{l}\text { Lower } \\
\text { Bound }\end{array}$ & $\begin{array}{l}\text { Upper } \\
\text { Bound }\end{array}$ \\
\hline \multirow{2}{*}{ Gender } & Male (ref.) & - & - & - & - & - & - & - & - & - \\
\hline & Female & $1.5585(0.004)$ & 1.1568 & 2.1000 & $2.1101(0.001)$ & 1.3342 & 3.3370 & $1.1018(0.758)$ & 0.5942 & 2.0430 \\
\hline \multirow{3}{*}{$\begin{array}{l}\text { Body mass } \\
\text { change }\end{array}$} & $\begin{array}{l}\text { No body } \\
\text { mass change } \\
\text { (ref.) }\end{array}$ & - & - & - & - & - & - & - & - & - \\
\hline & Lost weight & $0.7241(0.045)$ & 0.5279 & 0.9930 & $0.6353(0.096)$ & 0.3723 & 1.0840 & $1.0905(0.821)$ & 0.5158 & 2.3060 \\
\hline & $\begin{array}{l}\text { Gained } \\
\text { weight }\end{array}$ & $1.6521(0.003)$ & 1.1806 & 2.3120 & $\begin{array}{l}3.8277 \\
(<0.001)\end{array}$ & 2.4659 & 5.9420 & $\begin{array}{c}3.3635 \\
(<0.001)\end{array}$ & 1.7222 & 6.5690 \\
\hline \multirow{4}{*}{ Body mass } & $\begin{array}{c}\text { Normal } \\
\text { weight (ref.) }\end{array}$ & - & - & - & - & - & - & - & - & - \\
\hline & Malnourished & $0.6112(0.177)$ & 0.2990 & 1.2490 & $0.6008(0.422)$ & 0.1733 & 2.0820 & $\begin{array}{l}<0.001 \\
(<0.001)\end{array}$ & $<0.001$ & $<0.001$ \\
\hline & Overweight & $0.9656(0.864)$ & 0.6461 & 1.4430 & $1.8217(0.021)$ & 1.0929 & 3.0370 & $1.9995(0.051)$ & 0.9983 & 4.0050 \\
\hline & Obese & 2.1307 (0.009) & 1.2058 & 3.7650 & $\begin{array}{c}4.3035 \\
(<0.001)\end{array}$ & 2.2301 & 8.3050 & $1.7574(0.331)$ & 0.5637 & 5.4790 \\
\hline
\end{tabular}

OR-Odds Ratio.

The estimated effects of selected predictors using multinomial logistic regression for the declared frequency of confirmation for EEQ question 5 'Eating when they are stressed, angry or bored' in the PLACE-19 Study sample are presented in Table 6. The results of multinomial logistic regression indicated that declared frequency of confirmation of always eating when they are stressed, angry or bored was higher in respondents gaining weight while compared with those reporting no body mass change (OR 1.8540 (1.0528-3.2670); $p=0.032$ ), and in obese respondents while compared with normal weight ones (OR 3.1500 $(1.4518-6.8350 ; p=0.004)$.

Table 6. Estimated effects of selected predictors using multinomial logistic regression for the declared frequency of confirmation for Emotional Eater Questionnaire (EEQ) question 5 `Eating when they are stressed, angry or bored' in the PLACE-19 Study sample $(n=1126)$.

\begin{tabular}{|c|c|c|c|c|c|c|c|c|c|c|}
\hline \multirow{2}{*}{ Variable } & \multirow{2}{*}{ Categories } & \multirow{2}{*}{$\begin{array}{c}\text { Declared } \\
\text { 'Sometimes' } \\
\text { OR } \\
(p \text {-Value) }\end{array}$} & \multicolumn{2}{|c|}{$95 \%$ Confidence Interval } & \multirow{2}{*}{$\begin{array}{c}\text { Declared } \\
\text { 'Generally' } \\
\text { OR } \\
\text { ( } p \text {-Value) }\end{array}$} & \multicolumn{2}{|c|}{$\begin{array}{l}\text { 95\% Confidence } \\
\text { Interval }\end{array}$} & \multirow{2}{*}{$\begin{array}{c}\text { Declared } \\
\text { ‘Always' OR } \\
(p \text {-Value })\end{array}$} & \multicolumn{2}{|c|}{$\begin{array}{l}\text { 95\% Confidence } \\
\text { Interval }\end{array}$} \\
\hline & & & $\begin{array}{l}\text { Lower } \\
\text { Bound }\end{array}$ & $\begin{array}{l}\text { Upper } \\
\text { Bound }\end{array}$ & & $\begin{array}{l}\text { Lower } \\
\text { Bound }\end{array}$ & $\begin{array}{l}\text { Upper } \\
\text { Bound }\end{array}$ & & $\begin{array}{l}\text { Lower } \\
\text { Bound }\end{array}$ & $\begin{array}{l}\text { Upper } \\
\text { Bound }\end{array}$ \\
\hline \multirow[b]{2}{*}{ Gender } & Male (ref.) & - & - & - & - & - & - & - & - & - \\
\hline & Female & $1.3280(0.062)$ & 0.9860 & 1.7890 & $\begin{array}{c}2.5260 \\
(<0.001)\end{array}$ & 1.6256 & 3.9260 & $1.5440(0.126)$ & 0.8857 & 2.6900 \\
\hline \multirow{3}{*}{$\begin{array}{l}\text { Body mass } \\
\text { change }\end{array}$} & $\begin{array}{l}\text { No body } \\
\text { mass change } \\
\text { (ref.) }\end{array}$ & - & - & - & - & - & - & - & - & - \\
\hline & Lost weight & $0.8500(0.322)$ & 0.6166 & 1.1720 & $0.7370(0.196)$ & 0.4637 & 1.1710 & $0.6850(0.246)$ & 0.3607 & 1.2990 \\
\hline & $\begin{array}{l}\text { Gained } \\
\text { weight }\end{array}$ & $1.3540(0.085)$ & 0.9596 & 1.9100 & $\begin{array}{l}2.7570 \\
(<0.001)\end{array}$ & 1.8213 & 4.1730 & $1.8540(0.032)$ & 1.0528 & 3.2670 \\
\hline \multirow{4}{*}{ Body mass } & $\begin{array}{c}\text { Normal } \\
\text { weight (ref.) }\end{array}$ & - & - & - & - & - & - & - & - & - \\
\hline & Malnourished & $0.5040(0.069)$ & 0.2413 & 1.0550 & $0.4670(0.173)$ & 0.1560 & 1.3950 & $0.2960(0.239)$ & 0.0389 & 2.2450 \\
\hline & Overweight & $1.1740(0.427)$ & 0.7902 & 1.7450 & $1.0730(0.791)$ & 0.6363 & 1.8100 & $1.5740(0.180)$ & 0.8116 & 3.0520 \\
\hline & Obese & $1.5250(0.142)$ & 0.8683 & 2.6770 & $1.7450(0.101)$ & 0.8970 & 3.3950 & $3.1500(0.004)$ & 1.4518 & 6.8350 \\
\hline
\end{tabular}


The estimated effects of selected predictors using multinomial logistic regression for the declared frequency of confirmation for EEQ question 6 'Eating more of their favourite food and with less control when they are alone' in the PLACE-19 Study sample are presented in Table 7. The results of multinomial logistic regression indicated that declared frequency of confirmation of always eating more of their favourite food and with less control when they are alone was higher in female while compared with male respondents (OR 3.5735 (1.9036-6.7080); $p<0.001$ ), in respondents gaining weight while compared with those reporting no body mass change (OR 2.7144 (1.5990-4.6080); $p<0.001$ ), and in obese respondents while compared with normal weight ones (OR 2.7163 (1.1612-6.3540); $p<0.021)$.

Table 7. Estimated effects of selected predictors using multinomial logistic regression for the declared frequency of confirmation for Emotional Eater Questionnaire (EEQ) question 6 'Eating more of their favourite food and with less control when they are alone' in the PLACE-19 Study sample $(n=1126)$.

\begin{tabular}{|c|c|c|c|c|c|c|c|c|c|c|}
\hline \multirow{2}{*}{ Variable } & \multirow{2}{*}{ Categories } & \multirow{2}{*}{$\begin{array}{c}\text { Declared } \\
\text { 'Sometimes' } \\
\text { OR } \\
(p \text {-Value) }\end{array}$} & \multicolumn{2}{|c|}{ 95\% Confidence Interval } & \multirow{2}{*}{$\begin{array}{c}\text { Declared } \\
\text { 'Generally' } \\
\text { OR } \\
(p \text {-Value) }\end{array}$} & \multicolumn{2}{|c|}{$\begin{array}{l}\text { 95\% Confidence } \\
\text { Interval }\end{array}$} & \multirow{2}{*}{$\begin{array}{c}\text { Declared } \\
\text { 'Always' OR } \\
\text { ( } p \text {-Value })\end{array}$} & \multicolumn{2}{|c|}{$\begin{array}{l}\text { 95\% Confidence } \\
\text { Interval }\end{array}$} \\
\hline & & & $\begin{array}{l}\text { Lower } \\
\text { Bound }\end{array}$ & $\begin{array}{l}\text { Upper } \\
\text { Bound }\end{array}$ & & $\begin{array}{l}\text { Lower } \\
\text { Bound }\end{array}$ & $\begin{array}{l}\text { Upper } \\
\text { Bound }\end{array}$ & & $\begin{array}{l}\text { Lower } \\
\text { Bound }\end{array}$ & $\begin{array}{l}\text { Upper } \\
\text { Bound }\end{array}$ \\
\hline \multirow{2}{*}{ Gender } & Male (ref.) & - & - & - & - & - & - & - & - & - \\
\hline & Female & $1.5977(0.003)$ & 1.1775 & 2.1680 & $\begin{array}{c}2.2497 \\
(<0.001)\end{array}$ & 1.5072 & 3.3580 & $\begin{array}{c}3.5735 \\
(<0.001)\end{array}$ & 1.9036 & 6.7080 \\
\hline \multirow{3}{*}{$\begin{array}{l}\text { Body mass } \\
\text { change }\end{array}$} & $\begin{array}{l}\text { No body } \\
\text { mass change } \\
\text { (ref.) }\end{array}$ & - & - & - & - & - & - & - & - & - \\
\hline & Lost weight & $0.8798(0.445)$ & 0.6336 & 1.2220 & $1.0152(0.945)$ & 0.6614 & 1.5580 & $0.5706(0.097)$ & 0.2943 & 1.1060 \\
\hline & $\begin{array}{l}\text { Gained } \\
\text { weight }\end{array}$ & $1.2583(0.207)$ & 0.8810 & 1.7970 & $\begin{array}{c}2.9825 \\
(<0.001)\end{array}$ & 1.9861 & 4.4790 & $\begin{array}{c}2.7144 \\
(<0.001)\end{array}$ & 1.5990 & 4.6080 \\
\hline \multirow{4}{*}{ Body mass } & $\begin{array}{c}\text { Normal } \\
\text { weight (ref.) }\end{array}$ & - & - & - & - & - & - & - & - & - \\
\hline & Malnourished & $0.9040(0.790)$ & 0.4299 & 1.9010 & $0.8737(0.784)$ & 0.3330 & 2.2920 & $1.0937(0.891)$ & 0.3052 & 3.9200 \\
\hline & Overweight & $1.1825(0.416)$ & 0.7898 & 1.7700 & 1.0917 (0.729) & 0.6647 & 1.7930 & $1.2711(0.477)$ & 0.6560 & 2.4630 \\
\hline & Obese & $2.6021(0.002)$ & 1.4329 & 4.7260 & $2.2613(0.019)$ & 1.1406 & 4.4830 & $2.7163(0.021)$ & 1.1612 & 6.3540 \\
\hline
\end{tabular}

The estimated effects of selected predictors using multinomial logistic regression for the declared frequency of confirmation for EEQ question 7 'Feeling guilty when eating "forbidden" foods, like sweets or snacks' in the PLACE-19 Study sample are presented in Table 8 . The results of multinomial logistic regression indicated that declared frequency of confirmation of always feeling guilty when eating "forbidden" foods, like sweets or snacks was higher in female while compared with male respondents (OR 25.2110 (7.8750-80.7126); $p<0.001)$, in respondents gaining weight while compared with those reporting no body mass change (OR $2.2422(1.3628-3.6890) ; p=0.001)$, and in obese respondents while compared with normal weight ones (OR $2.3796(1.1180-5.0650) ; p=0.024)$. 
Table 8. Estimated effects of selected predictors using multinomial logistic regression for the declared frequency of confirmation for Emotional Eater Questionnaire (EEQ) question 7 'Feeling guilty when eating "forbidden" foods, like sweets or snacks' in the PLACE-19 Study sample $(n=1126)$.

\begin{tabular}{|c|c|c|c|c|c|c|c|c|c|c|}
\hline \multirow{2}{*}{ Variable } & \multirow{2}{*}{ Categories } & \multirow{2}{*}{$\begin{array}{c}\text { Declared } \\
\text { 'Sometimes' } \\
\text { OR } \\
(p \text {-Value) }\end{array}$} & \multicolumn{2}{|c|}{ 95\% Confidence Interval } & \multirow{2}{*}{$\begin{array}{c}\text { Declared } \\
\text { ‘Generally' } \\
\text { OR } \\
(p \text {-Value) }\end{array}$} & \multicolumn{2}{|c|}{$\begin{array}{c}\text { 95\% Confidence } \\
\text { Interval }\end{array}$} & \multirow{2}{*}{$\begin{array}{c}\text { Declared } \\
\text { 'Always' OR } \\
\text { ( } p \text {-value })\end{array}$} & \multicolumn{2}{|c|}{$\begin{array}{l}\text { 95\% Confidence } \\
\text { Interval }\end{array}$} \\
\hline & & & $\begin{array}{l}\text { Lower } \\
\text { Bound }\end{array}$ & $\begin{array}{l}\text { Upper } \\
\text { Bound }\end{array}$ & & $\begin{array}{l}\text { Lower } \\
\text { Bound }\end{array}$ & $\begin{array}{l}\text { Upper } \\
\text { Bound }\end{array}$ & & $\begin{array}{l}\text { Lower } \\
\text { Bound }\end{array}$ & $\begin{array}{l}\text { Upper } \\
\text { Bound }\end{array}$ \\
\hline \multirow[b]{2}{*}{ Gender } & Male (ref.) & - & - & - & - & - & - & - & - & - \\
\hline & Female & $1.6501(0.001)$ & 1.2117 & 2.2473 & $\begin{array}{l}3.1465 \\
(<0.001) \\
\end{array}$ & 2.0285 & 4.8807 & $\begin{array}{l}25.2110 \\
(<0.001)\end{array}$ & 7.8750 & 80.7126 \\
\hline \multirow{3}{*}{$\begin{array}{l}\text { Body mass } \\
\text { change }\end{array}$} & $\begin{array}{l}\text { No body } \\
\text { mass change } \\
\text { (ref.) }\end{array}$ & - & - & - & - & - & - & - & - & - \\
\hline & Lost weight & $1.1959(0.316)$ & 0.8431 & 1.6962 & $1.5927(0.030)$ & 1.0452 & 2.4270 & $1.7169(0.036)$ & 1.0346 & 2.8494 \\
\hline & $\begin{array}{l}\text { Gained } \\
\text { weight }\end{array}$ & $1.3417(0.095)$ & 0.9504 & 1.8940 & $1.6101(0.029)$ & 1.0506 & 2.4677 & $2.2422(0.001)$ & 1.3628 & 3.6890 \\
\hline \multirow{4}{*}{ Body mass } & $\begin{array}{c}\text { Normal } \\
\text { weight (ref.) }\end{array}$ & - & - & - & - & - & - & - & - & - \\
\hline & Malnourished & $0.6230(0.242)$ & 0.2821 & 1.3759 & $0.6596(0.416)$ & 0.2420 & 1.7976 & $0.3873(0.211)$ & 0.0876 & 1.7128 \\
\hline & Overweight & $1.1464(0.523)$ & 0.7541 & 1.7427 & $1.4301(0.162)$ & 0.8666 & 2.3600 & $1.5800(0.119)$ & 0.8896 & 2.8061 \\
\hline & Obese & $1.8114(0.047)$ & 1.0077 & 3.2561 & $\begin{array}{l}3.10935 \\
(<0.001)\end{array}$ & 1.6639 & 5.8100 & $2.3796(0.024)$ & 1.1180 & 5.0650 \\
\hline
\end{tabular}

OR-Odds Ratio.

The estimated effects of selected predictors using multinomial logistic regression for the declared frequency of confirmation for EEQ question 8 'Feeling less control over their diet when they are tired after work at night' in the PLACE-19 Study sample are presented in Table 9. The results of multinomial logistic regression indicated that declared frequency of confirmation of always feeling less control over their diet when they are tired after work at night was higher in respondents gaining weight while compared with those reporting no body mass change (OR $2.6434(1.4697-4.7540) ; p=0.001)$.

Table 9. Estimated effects of selected predictors using multinomial logistic regression for the declared frequency of confirmation for Emotional Eater Questionnaire (EEQ) question 8 'Feeling less control over their diet when they are tired after work at night' in the PLACE-19 Study sample $(n=1126)$.

\begin{tabular}{|c|c|c|c|c|c|c|c|c|c|c|}
\hline \multirow{2}{*}{ Variable } & \multirow{2}{*}{ Categories } & \multirow{2}{*}{$\begin{array}{c}\text { Declared } \\
\text { 'Sometimes' } \\
\text { OR } \\
(p \text {-Value) }\end{array}$} & \multicolumn{2}{|c|}{ 95\% Confidence Interval } & \multirow{2}{*}{$\begin{array}{c}\text { Declared } \\
\text { 'Generally' } \\
\text { OR } \\
(p \text {-Value })\end{array}$} & \multicolumn{2}{|c|}{$\begin{array}{c}95 \% \text { Confidence } \\
\text { Interval }\end{array}$} & \multirow{2}{*}{$\begin{array}{c}\text { Declared } \\
\text { ‘Always' OR } \\
(p \text {-Value })\end{array}$} & \multicolumn{2}{|c|}{$\begin{array}{l}\text { 95\% Confidence } \\
\text { Interval }\end{array}$} \\
\hline & & & $\begin{array}{l}\text { Lower } \\
\text { Bound }\end{array}$ & $\begin{array}{l}\text { Upper } \\
\text { Bound }\end{array}$ & & $\begin{array}{l}\text { Lower } \\
\text { Bound }\end{array}$ & $\begin{array}{l}\text { Upper } \\
\text { Bound }\end{array}$ & & $\begin{array}{l}\text { Lower } \\
\text { Bound }\end{array}$ & $\begin{array}{l}\text { Upper } \\
\text { Bound }\end{array}$ \\
\hline \multirow{2}{*}{ Gender } & Male (ref.) & - & - & - & - & - & - & - & - & - \\
\hline & Female & $1.0994(0.537)$ & 0.8135 & 1.4860 & $2.0076(0.002)$ & 1.2940 & 3.1150 & $1.5007(0.169)$ & 0.8414 & 2.6760 \\
\hline \multirow{3}{*}{$\begin{array}{l}\text { Body mass } \\
\text { change }\end{array}$} & $\begin{array}{l}\text { No body } \\
\text { mass change } \\
\text { (ref.) }\end{array}$ & - & - & - & - & - & - & - & - & - \\
\hline & Lost weight & $1.0224(0.897)$ & 0.7325 & 1.4270 & $1.1345(0.577)$ & 0.7280 & 1.7680 & $1.4963(0.193)$ & 0.8152 & 2.7460 \\
\hline & $\begin{array}{l}\text { Gained } \\
\text { weight }\end{array}$ & $\begin{array}{c}1.7637 \\
(<0.001)\end{array}$ & 1.2621 & 2.4650 & $\begin{array}{c}2.4655 \\
(<0.001)\end{array}$ & 1.6207 & 3.7510 & $2.6434(0.001)$ & 1.4697 & 4.7540 \\
\hline \multirow{4}{*}{ Body mass } & $\begin{array}{c}\text { Normal } \\
\text { weight (ref.) }\end{array}$ & - & - & - & - & - & - & - & - & - \\
\hline & Malnourished & $0.4246(0.049)$ & 0.1812 & 0.9950 & $0.4060(0.148)$ & 0.1199 & 1.3750 & $0.9451(0.929)$ & 0.2743 & 3.2560 \\
\hline & Overweight & $1.0948(0.657)$ & 0.7341 & 1.6330 & $1.3002(0.298)$ & 0.7927 & 2.1330 & $1.2863(0.471)$ & 0.6484 & 2.5520 \\
\hline & Obese & $2.0593(0.008)$ & 1.2072 & 3.5130 & $1.7693(0.096)$ & 0.9042 & 3.4620 & $2.0452(0.102)$ & 0.8683 & 4.8170 \\
\hline
\end{tabular}

OR-Odds Ratio.

The estimated effects of selected predictors using multinomial logistic regression for the declared frequency of confirmation for EEQ question 9 'Giving up and starting eating without control, particularly food that they think is fattening, after overeating while on a

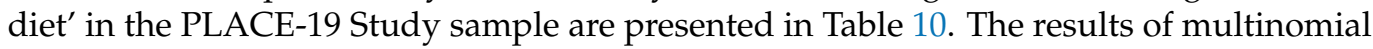
logistic regression indicated that declared frequency of confirmation of always giving up 
and starting eating without control, particularly food that they think is fattening, after overeating while on a diet was higher in female while compared with male respondents (OR 3.6500 (1.6062-8.2942); $p=0.002$ ), and in respondents gaining weight while compared with those reporting no body mass change (OR $4.4987(2.3704-8.5380) ; p<0.001)$.

Table 10. Estimated effects of selected predictors using multinomial logistic regression for the declared frequency of confirmation for Emotional Eater Questionnaire (EEQ) question 9 'Giving up and starting eating without control, particularly food that they think is fattening, after overeating while on a diet' in the PLACE-19 Study sample $(n=1126)$.

\begin{tabular}{|c|c|c|c|c|c|c|c|c|c|c|}
\hline \multirow{2}{*}{ Variable } & \multirow{2}{*}{ Categories } & \multirow{2}{*}{$\begin{array}{c}\text { Declared } \\
\text { 'Sometimes' } \\
\text { OR } \\
(p \text {-Value })\end{array}$} & \multicolumn{2}{|c|}{ 95\% Confidence Interval } & \multirow{2}{*}{$\begin{array}{c}\text { Declared } \\
\text { ‘Generally' } \\
\text { OR } \\
(p \text {-Value) }\end{array}$} & \multicolumn{2}{|c|}{$\begin{array}{l}\text { 95\% Confidence } \\
\text { Interval }\end{array}$} & \multirow{2}{*}{$\begin{array}{c}\text { Declared } \\
\text { 'Always' OR } \\
\text { ( } p \text {-Value })\end{array}$} & \multicolumn{2}{|c|}{$\begin{array}{l}\text { 95\% Confidence } \\
\text { Interval }\end{array}$} \\
\hline & & & $\begin{array}{l}\text { Lower } \\
\text { Bound }\end{array}$ & $\begin{array}{l}\text { Upper } \\
\text { Bound }\end{array}$ & & $\begin{array}{l}\text { Lower } \\
\text { Bound }\end{array}$ & $\begin{array}{l}\text { Upper } \\
\text { Bound }\end{array}$ & & $\begin{array}{l}\text { Lower } \\
\text { Bound }\end{array}$ & $\begin{array}{l}\text { Upper } \\
\text { Bound }\end{array}$ \\
\hline \multirow[b]{2}{*}{ Gender } & Male (ref.) & - & - & - & - & - & - & - & - & - \\
\hline & Female & $1.3623(0.050)$ & 0.9995 & 1.8568 & $\begin{array}{c}2.8575 \\
(<0.001)\end{array}$ & 1.6820 & 4.8545 & $3.6500(0.002)$ & 1.6062 & 8.2942 \\
\hline \multirow{3}{*}{$\begin{array}{l}\text { Body mass } \\
\text { change }\end{array}$} & $\begin{array}{l}\text { No body } \\
\text { mass change } \\
\text { (ref.) }\end{array}$ & - & - & - & - & - & - & - & - & - \\
\hline & Lost weight & $1.0920(0.607)$ & 0.7813 & 1.5262 & $0.7759(0.359)$ & 0.4513 & 1.3338 & $0.8274(0.656)$ & 0.3596 & 1.9042 \\
\hline & $\begin{array}{l}\text { Gained } \\
\text { weight }\end{array}$ & $\begin{array}{c}2.1135 \\
(<0.001)\end{array}$ & 1.5106 & 2.9569 & $\begin{array}{c}2.9017 \\
(<0.001)\end{array}$ & 1.8397 & 4.5768 & $\begin{array}{c}4.4987 \\
(<0.001)\end{array}$ & 2.3704 & 8.5380 \\
\hline \multirow{4}{*}{ Body mass } & $\begin{array}{c}\text { Normal } \\
\text { weight (ref.) }\end{array}$ & - & - & - & - & - & - & - & - & - \\
\hline & Malnourished & $0.5897(0.196)$ & 0.2646 & 1.3138 & $\begin{array}{l}<0.001 \\
(<0.001)\end{array}$ & $<0.001$ & $<0.001$ & $0.4955(0.500)$ & 0.0645 & 3.8074 \\
\hline & Overweight & $1.0203(0.923)$ & 0.6805 & 1.5297 & $1.2986(0.356)$ & 0.7453 & 2.2627 & $1.3592(0.425)$ & 0.6399 & 2.8871 \\
\hline & Obese & $2.2872(0.002)$ & 1.3434 & 3.8941 & $\begin{array}{c}3.4171 \\
(<0.001)\end{array}$ & 1.7869 & 6.5348 & $2.2080(0.108)$ & 0.8405 & 5.8007 \\
\hline
\end{tabular}

OR-Odds Ratio.

The estimated effects of selected predictors using multinomial logistic regression for the declared frequency of confirmation for EEQ question 10 'Feeling that food controls them, rather than they control food' in the PLACE-19 Study sample are presented in Table 11. The results of multinomial logistic regression indicated that declared frequency of confirmation of always feeling that food controls them, rather than they control food was higher in female while compared with male respondents (OR 3.8449 (1.4827-9.9709); $p=0.006$ ), in respondents gaining weight while compared with those reporting no body mass change (OR 3.8514 (1.8006-8.2378); $p<0.001)$, and in obese respondents while compared with normal weight ones (OR $3.0463(1.0712-8.6629) ; p=0.037)$. 
Table 11. Estimated effects of selected predictors using multinomial logistic regression for the declared frequency of confirmation for Emotional Eater Questionnaire (EEQ) question 10 'Feeling that food controls them, rather than they control food' in the PLACE-19 Study sample $(n=1126)$.

\begin{tabular}{|c|c|c|c|c|c|c|c|c|c|c|}
\hline \multirow{2}{*}{ Variable } & \multirow{2}{*}{ Categories } & \multirow{2}{*}{$\begin{array}{c}\text { Declared } \\
\text { 'Sometimes' } \\
\text { OR } \\
(p \text {-Value) }\end{array}$} & \multicolumn{2}{|c|}{$95 \%$ Confidence Interval } & \multirow{2}{*}{$\begin{array}{c}\text { Declared } \\
\text { ‘Generally' } \\
\text { OR } \\
(p \text {-Value })\end{array}$} & \multicolumn{2}{|c|}{$\begin{array}{l}\text { 95\% Confidence } \\
\text { Interval }\end{array}$} & \multirow{2}{*}{$\begin{array}{c}\text { Declared } \\
\text { 'Always' OR } \\
(p \text {-Value })\end{array}$} & \multicolumn{2}{|c|}{$\begin{array}{l}\text { 95\% Confidence } \\
\text { Interval }\end{array}$} \\
\hline & & & $\begin{array}{l}\text { Lower } \\
\text { Bound }\end{array}$ & $\begin{array}{l}\text { Upper } \\
\text { Bound }\end{array}$ & & $\begin{array}{l}\text { Lower } \\
\text { Bound }\end{array}$ & $\begin{array}{l}\text { Upper } \\
\text { Bound }\end{array}$ & & $\begin{array}{l}\text { Lower } \\
\text { Bound }\end{array}$ & $\begin{array}{l}\text { Upper } \\
\text { Bound }\end{array}$ \\
\hline \multirow[b]{2}{*}{ Gender } & Male (ref.) & - & - & - & - & - & - & - & - & - \\
\hline & Female & $1.3288(0.080)$ & 0.9665 & 1.8270 & $\begin{array}{l}2.3278 \\
(<0.001)\end{array}$ & 1.4257 & 3.8007 & $3.8449(0.006)$ & 1.4827 & 9.9709 \\
\hline \multirow{3}{*}{$\begin{array}{l}\text { Body mass } \\
\text { change }\end{array}$} & $\begin{array}{c}\text { No body } \\
\text { mass change } \\
\text { (ref.) }\end{array}$ & - & - & - & - & - & - & - & - & - \\
\hline & Lost weight & $0.9408(0.737)$ & 0.6593 & 1.3426 & $1.359(0.230)$ & 0.8238 & 2.2420 & $2.0469(0.075)$ & 0.9313 & 4.4990 \\
\hline & $\begin{array}{l}\text { Gained } \\
\text { weight }\end{array}$ & $\begin{array}{c}2.1139 \\
(<0.001)\end{array}$ & 1.5093 & 2.9606 & $\begin{array}{c}3.6273 \\
(<0.001)\end{array}$ & 2.2997 & 5.7213 & $\begin{array}{c}3.8514 \\
(<0.001)\end{array}$ & 1.8006 & 8.2378 \\
\hline \multirow{4}{*}{ Body mass } & $\begin{array}{c}\text { Normal } \\
\text { weight (ref.) }\end{array}$ & - & - & - & - & - & - & - & - & - \\
\hline & Malnourished & $0.313(0.031)$ & 0.1090 & 0.8989 & $0.4111(0.232)$ & 0.0956 & 1.7683 & $\begin{array}{l}<0.0001 \\
(0.973)\end{array}$ & $<0.001$ & $<0.001$ \\
\hline & Overweight & $1.2446(0.295)$ & 0.8263 & 1.8746 & $1.7329(0.040)$ & 1.0245 & 2.9311 & $2.0413(0.068)$ & 0.9497 & 4.3874 \\
\hline & Obese & $\begin{array}{c}2.8602 \\
(<0.001)\end{array}$ & 1.6264 & 5.0301 & $\begin{array}{c}5.9320 \\
(<0.001)\end{array}$ & 3.2078 & 10.9698 & $3.0463(0.037)$ & 1.0712 & 8.6629 \\
\hline
\end{tabular}

OR-Odds Ratio.

\section{Discussion}

Emotional eating is considered as a coping mechanism during stressful moments, and is based on some typical eating behaviors [43]. Although it has been well established that decreased appetite is commonly observed among children in relation to stress [44], the intensity of emotional eating significantly increases during the period between childhood and adolescence [45]. As perceived stress and the associated emotional eating behavior may lead to higher consumption of sugar- and fat-rich foods [46], they are identified as risk factors for the development of obesity [47]. A number of studies conducted on adults have confirmed this relationship, as emotional eating was found to be associated with an increased BMI [48-50]. However, no study has so far proven such dependency for children or adolescents, and the results are inconclusive [4]. The study by Nguyen-Rodriguez et al. [21] performed on American middle school students showed that no differences in emotional eating were noted between normal weight and overweight adolescents. However, the study by Braet \& van Strien [51] conducted on Belgian children found that overweight and obese participants scored significantly higher for emotional eating compared to children with a normal weight. At the same time, the study by Croker et al. [52] showed that the prevalence of emotional eating was the highest among obese children, compared to underweight, normal weight, and overweight participants. Similar results were observed in the study by Geliebter \& Aversa [53], in which underweight participants reported that they eat less during negative emotional states whereas overweight participants reported that they overeat while experiencing negative emotions.

The present study showed that a higher share of emotional eaters and very emotional eaters were found among overweight and obese respondents compared to the other groups. Similarly, obese respondents more often declared a number of emotional eating behaviours. Other studies also report that emotional eating is often connected to craving for chocolates [54] and that individuals with an excessive body mass, who aim to reduce their body weight, try to avoid eating this product, as it is considered as forbidden and high in calories, while its consumption results in guilt and high dietary restrictions, as well as a negative attitude toward one's own body $[55,56]$.

Gender has been identified as a key biological determinant of emotional eating [57]. In the present study, female respondents were found to be more prone to emotional eating, compared to males. This result is in compliance with other studies $[48,58,59]$ which have 
indicated that women are especially susceptible to eating in response to negative emotions. The study of Péneau et al. [48] highlighted that French adult women more likely engaged in emotional eating than men. Similarly, the study by Sze et al. [58], which was carried out among Chinese university students, revealed that women were characterized by about threefold higher likelihood of emotional eating than men. Additionally, in the study by Camilleri et al. [59], which analyzed the association between emotional eating and intake of energy-dense snacks, such association was observed to be stronger in women with depressive symptoms, compared to men with depressive symptoms. The vulnerability of women to emotional eating can be explained by changes in the level of ovarian hormones across the menstrual cycle [60] and the specific moderator effect of 5-HTTLPR genotype [61]. It should also be borne in mind that emotional eating is linked with an increased risk of eating disorders, such as binge eating [62], which is more frequent among women than men [63].

The findings of our study indicate that the issue of emotional eating should be addressed in high-risk groups, such as female and excessive body weight adolescents. While females may be considered as especially prone to emotional eating, excessive body mass may be treated as a consequence. Special attention should be paid to female adolescents with an excessive body mass while addressing emotional eating in general public health policy. Although some important observations were formulated in the studied group, an association was observed between gender and body mass change during the COVID-19 pandemic, which may be mentioned as a source of bias within the study and may have interfered with the results. Therefore, further studies, especially prospective ones, are needed to analyze the relationship between emotional eating and body mass change. Moreover, self-report bias must be indicated, both for body mass and emotional eating behaviors, which especially in case of body mass, may be a serious problem, as participants may underestimate or overestimate their body mass. However, it is a common problem in population-based studies, but if the aim of the study is to assess the large sample from the whole country and representative for all the regions, this bias often must be accepted, as simplified model of compromised accuracy is chosen [31].

\section{Conclusions}

Thus, it may be concluded that among Polish adolescents gender, body mass, and body mass change during the COVID-19 pandemic are the major determinants of emotional eating behaviors and that female individuals, obese individuals, and those gaining weight are particularly more vulnerable to emotional eating behaviors than others. Therefore, emotional eating should be addressed in general public health policy and appropriate education should be provided to vulnerable groups such as female and obese adolescents.

Author Contributions: D.S., D.G. (Dominika Głąbska), D.G. (Dominika Guzek) made study conception and design; D.S., D.G. (Dominika Głabska), D.G. (Dominika Guzek) performed the research; D.S., D.G. (Dominika Głąbska), D.G. (Dominika Guzek) analyzed the data; D.S., D.G. (Dominika Głabska), D.G. (Dominika Guzek) interpreted the data; D.S., D.G. (Dominika Głabska), D.G. (Dominika Guzek) wrote the paper. All authors have read and agreed to the published version of the manuscript.

Funding: This research was funded by the Polish Ministry of Science and Higher Education within funds of Institute of Human Nutrition Sciences, Warsaw University of Life Sciences (WULS), for scientific research.

Institutional Review Board Statement: The study was carried out in accordance with the guidelines laid down in the Declaration of Helsinki and all procedures involving human subjects received the approval of the Ethics Committee of the Central Clinical Hospital of the Ministry of Interior and Administration in Warsaw (No. 2/2021).

Informed Consent Statement: Informed consent was obtained from all subjects involved in the study.

Conflicts of Interest: The authors declare no conflict of interest. 


\section{References}

1. Konttinen, H. Emotional eating and obesity in adults: The role of depression, sleep and genes. Proc. Nutr. Soc. 2020, 79, 283-289. [CrossRef]

2. Macht, M. How emotions affect eating: A five-way model. Appetite 2008, 50, 1-11. [CrossRef] [PubMed]

3. Frayn, M.; Livshits, S.; Knäuper, B. Emotional eating and weight regulation: A qualitative study of compensatory behaviors and concerns. J. Eat. Disord. 2018, 6, 23. [CrossRef]

4. Jalo, E.; Konttinen, H.; Vepsäläinen, H.; Chaput, J.-P.; Hu, G.; Maher, C.; Maia, J.; Sarmiento, O.L.; Standage, M.; Tudor-Locke, C.; et al. Emotional Eating, Health Behaviours, and Obesity in Children: A 12-Country Cross-Sectional Study. Nutrients 2019, 11, 351. [CrossRef] [PubMed]

5. Frayn, M.; Knäuper, B. Emotional Eating and Weight in Adults: A Review. Curr. Psychol. 2017, 37, 924-933. [CrossRef]

6. Limbers, C.; Summers, E. Emotional Eating and Weight Status in Adolescents: A Systematic Review. Int. J. Environ. Res. Public Health 2021, 18, 991. [CrossRef] [PubMed]

7. Serafini, G.; Parmigiani, B.; Amerio, A.; Aguglia, A.; Sher, L.; Amore, M. The psychological impact of COVID-19 on the mental health in the general population. QJM 2020, 113, 531-537. [CrossRef]

8. Hill, D.C.; Moss, R.H.; Sykes-Muskett, B.; Conner, M.; O'Connor, D.B. Stress and eating behaviors in children and adolescents: Systematic review and meta-analysis. Appetite 2018, 123, 14-22. [CrossRef] [PubMed]

9. Rodríguez-Hidalgo, A.J.; Pantaleón, Y.; Dios, I.; Falla, D. Fear of COVID-19, Stress, and Anxiety in University Undergraduate Students: A Predictive Model for Depression. Front. Psychol. 2020, 11, 591797. [CrossRef]

10. Yaribeygi, H.; Panahi, Y.; Sahraei, H.; Johnston, T.P.; Sahebkar, A. The impact of stress on body function: A review. EXCLI J. 2017, 16, 1057-1072. [CrossRef]

11. Doğan, T.; Tekin, E.G.; Katrancığlu, A. Feeding your feelings: A self-report measure of emotional eating. Procedia Soc. Behav. Sci. 2011, 15, 2074-2077. [CrossRef]

12. Meule, A.; Reichenberger, J.; Blechert, J. Development and Preliminary Validation of the Salzburg Emotional Eating Scale. Front. Psychol. 2018, 9, 88. [CrossRef] [PubMed]

13. Masheb, R.M.; Grilo, C.M. Emotional overeating and its associations with eating disorder psychopathology among overweight patients with Binge eating disorder. Int. J. Eat. Disord. 2005, 39, 141-146. [CrossRef] [PubMed]

14. Garaulet, M.; Canteras, M.; Morales, E.; López-Guimera, G.; Sánchez-Carracedo, D.; Corbalán-Tutau, M.D. Validation of a questionnaire on emotional eating for use in cases of obesity: The Emotional Eater Questionnaire (EEQ). Nutr. Hosp. 2012, 27, 645-651. [CrossRef]

15. Özcan, B.A.; Yeşillkaya, B. Adverse Effect of Emotional Eating Developed During the COVID-19 Pandemic on Healthy Nutrition, a Vicious Circle: A cross-sectional descriptive study. Rev. Española Nutr. Hum. Dietética 2021, 25, 1144. [CrossRef]

16. López-Moreno, M.; López, M.; Miguel, M.; Garcés-Rimón, M. Physical and Psychological Effects Related to Food Habits and Lifestyle Changes Derived from COVID-19 Home Confinement in the Spanish Population. Nutrients 2020, 12, 3445. [CrossRef]

17. United Nations Children's Fund. The Impact of COVID-19 on Children's Mental Health. Available online: https://www.unicef. org/india/impact-covid-19-childrens-mental-health (accessed on 2 August 2021).

18. Singh, S.; Roy, D.; Sinha, K.; Parveen, S.; Sharma, G.; Joshi, G. Impact of COVID-19 and lockdown on mental health of children and adolescents: A narrative review with recommendations. Psychiatry Res. 2020, 293, 113429. [CrossRef]

19. Philippe, K.; Chabanet, C.; Issanchou, S.; Monnery-Patris, S. Child eating behaviors, parental feeding practices and food shopping motivations during the COVID-19 lockdown in France: (How) did they change? Appetite 2021, 161, 105132. [CrossRef]

20. Braet, C.; Claus, L.; Goossens, L.; Moens, E.; Van Vlierberghe, L.; Soetens, B. Differences in Eating Style between Overweight and Normal-Weight Youngsters. J. Health Psychol. 2008, 13, 733-743. [CrossRef]

21. Nguyen-Rodriguez, S.T.; Chou, C.-P.; Unger, J.B.; Spruijt-Metz, D. BMI as a moderator of perceived stress and emotional eating in adolescents. Eat. Behav. 2008, 9, 238-246. [CrossRef]

22. Głabska, D.; Skolmowska, D.; Guzek, D. Population-Based Study of the Influence of the COVID-19 Pandemic on Hand Hygiene Behaviors-Polish Adolescents' COVID-19 Experience (PLACE-19) Study. Sustainability 2020, 12, 4930. [CrossRef]

23. Guzek, D.; Skolmowska, D.; Głąbska, D. Analysis of Gender-Dependent Personal Protective Behaviors in a National Sample: Polish Adolescents' COVID-19 Experience (PLACE-19) Study. Int. J. Environ. Res. Public Health 2020, 17, 5770. [CrossRef] [PubMed]

24. Skolmowska, D.; Głąbska, D.; Guzek, D. Hand Hygiene Behaviors in a Representative Sample of Polish Adolescents in Regions Stratified by COVID-19 Morbidity and by Confounding Variables (PLACE-19 Study): Is There Any Association? Pathogens 2020, 9, 1011. [CrossRef] [PubMed]

25. Głabska, D.; Skolmowska, D.; Guzek, D. Population-Based Study of the Changes in the Food Choice Determinants of Secondary School Students: Polish Adolescents' COVID-19 Experience (PLACE-19) Study. Nutrients 2020, 12, 2640. [CrossRef] [PubMed]

26. Guzek, D.; Skolmowska, D.; Głąbska, D. Appetitive Traits in a Population-Based Study of Polish Adolescents within the PLACE-19 Study: Validation of the Adult Eating Behavior Questionnaire. Nutrients 2020, 12, 3889. [CrossRef]

27. Skolmowska, D.; Głąbska, D.; Guzek, D. Differences in Adolescents' Food Habits Checklist (AFHC) Scores before and during Pandemic in a Population-Based Sample: Polish Adolescents' COVID-19 Experience (PLACE-19) Study. Nutrients 2021, 13, 1663. [CrossRef] 
28. Guzek, D.; Skolmowska, D.; Głąbska, D. Associations between Food Preferences, Food Approach, and Food Avoidance in a Polish Adolescents' COVID-19 Experience (PLACE-19) Study Population. Nutrients 2021, 13, 2427. [CrossRef]

29. Głąsska, D.; Skolmowska, D.; Guzek, D. Food Preferences and Food Choice Determinants in a Polish Adolescents' COVID-19 Experience (PLACE-19) Study. Nutrients 2021, 13, 2491. [CrossRef]

30. Skolmowska, D.; Głabska, D.; Guzek, D. Association between Food Preferences and Food Habits in a Polish Adolescents' COVID-19 Experience (PLACE-19) Study. Nutrients 2021, 13, 3003. [CrossRef]

31. Skolmowska, D.; Głąbska, D. Analysis of Heme and Non-Heme Iron Intake and Iron Dietary Sources in Adolescent Menstruating Females in a National Polish Sample. Nutrients 2019, 11, 1049. [CrossRef]

32. Kołota, A.; Głabska, D. COVID-19 Pandemic and Remote Education Contributes to Improved Nutritional Behaviors and Increased Screen Time in a Polish Population-Based Sample of Primary School Adolescents: Diet and Activity of Youth during COVID-19 (DAY-19) Study. Nutrients 2021, 13, 1596. [CrossRef] [PubMed]

33. The Central Statistical Office in Poland December 2019. Available online: https://bdl.stat.gov.pl/BDL/dane/podgrup/temat (accessed on 1 December 2021).

34. The Central Statistical Office in Poland December 2020. Available online: http://demografia.stat.gov.pl/bazademografia/Tables. aspx (accessed on 1 December 2021).

35. Polish Ministry of National Education. Available online: https://rspo.men.gov.pl/ (accessed on 1 December 2021).

36. Nuttall, F.Q. Body Mass Index: Obesity, BMI, and health: A critical review. Nutr. Today 2015, 50, 117-128. [CrossRef] [PubMed]

37. Kulaga, Z.; Litwin, M.; Tkaczyk, M.; Rózdzyńska, A.; Barwicka, K.; Grajda, A.; Swiader, A.; Gurzkowska, B.; Napieralska, E.; Pan, H. The height-, weight-, and BMI-for-age of Polish school-aged children and adolescents relative to international and local growth references. BMC Public Health 2010, 10, 109. [CrossRef] [PubMed]

38. OLAF Calculator from OLAF Study. Available online: http:/ / olaf.czd.pl/index.php?option=com_content\&view=article\&id=103: kalkulator (accessed on 16 September 2021).

39. De Onis, M.; Onyango, A.W.; Borghi, E.; Siyam, A.; Nishida, C.; Siekmann, J. Development of a WHO growth reference for school-aged children and adolescents. Bull. World Health Organ. 2007, 85, 660-667. [CrossRef] [PubMed]

40. Głąbska, D.; Guzek, D.; Mellová, B.; Zadka, K.; Żywczyk, K.; Gutkowska, K. The National After-School Athletics Program Participation as a Tool to Reduce the Risk of Obesity in Adolescents after One Year of Intervention: A Nationwide Study. Int. J. Environ. Res. Public Health 2019, 16, 405. [CrossRef] [PubMed]

41. World Health Organization. Body Mass Index-BMI. Available online: https://www.euro.who.int/en/health-topics/diseaseprevention/nutrition/a-healthy-lifestyle/body-mass-index-bmi (accessed on 16 September 2021).

42. Polish Ministry of National Education. Available online: https://www.gov.pl/web/edukacja/zawieszenie-zajec-w-szkolach (accessed on 16 September 2021).

43. Huang, J.; Wang, Y.; Ye, N.; Xu, X. Perceived Stress and Emotional Eating in Adolescence: Mediation through Negative-Focused Cognitive Emotion Regulation and Reward Sensitivity. 2020. Available online: https://doi.org/10.21203/rs.3.rs-133643/v1 (accessed on 12 January 2022).

44. Van Strien, T.; Oosterveld, P. The children's DEBQ for assessment of restrained, emotional, and external eating in 7- to 12-year-old children. Int. J. Eat. Disord. 2008, 41, 72-81. [CrossRef]

45. Van Strien, T. Causes of Emotional Eating and Matched Treatment of Obesity. Curr. Diabetes Rep. 2018, 18, 35. [CrossRef]

46. Cotter, E.W.; Kelly, N.R. Stress-related eating, mindfulness, and obesity. Health Psychol. 2018, 37, 516-525. [CrossRef]

47. Jáuregui-Lobera, I.; Montes-Martínez, M. Emotional Eating and Obesity. In Psychosomatic Medicine; IntechOpen: London, UK, 2020. [CrossRef]

48. Péneau, S.; Ménard, E.; Méjean, C.; Bellisle, F.; Hercberg, S. Sex and dieting modify the association between emotional eating and weight status. Am. J. Clin. Nutr. 2013, 97, 1307-1313. [CrossRef]

49. Keskitalo, K.; Tuorila, H.; Spector, T.D.; Cherkas, L.F.; Knaapila, A.; Kaprio, J.; Silventoinen, K.; Perola, M. The Three-Factor Eating Questionnaire, body mass index, and responses to sweet and salty fatty foods: A twin study of genetic and environmental associations. Am. J. Clin. Nutr. 2008, 88, 263-271. [CrossRef]

50. Konttinen, H.; Silventoinen, K.; Sarlio-Lähteenkorva, S.; Männistö, S.; Haukkala, A. Emotional eating and physical activity self-efficacy as pathways in the association between depressive symptoms and adiposity indicators. Am. J. Clin. Nutr. 2010, 92, 1031-1039. [CrossRef] [PubMed]

51. Braet, C.; Van Strien, T. Assessment of emotional, externally induced and restrained eating behaviour in nine to twelve-year-old obese and non-obese children. Behav. Res. Ther. 1997, 35, 863-873. [CrossRef]

52. Croker, H.; Cooke, L.; Wardle, J. Appetitive behaviours of children attending obesity treatment. Appetite 2011, 57, 525-529. [CrossRef] [PubMed]

53. Geliebter, A.; Aversa, A. Emotional eating in overweight, normal weight, and underweight individuals. Eat. Behav. 2003, 3 , 341-347. [CrossRef]

54. Brytek-Matera, A.; Czepczor-Bernat, K.; Olejniczak, D. Food-related behaviours among individuals with overweight/obesity and normal body weight. Nutr. J. 2018, 17, 93. [CrossRef]

55. O'Reilly, G.A.; Cook, L.; Spruijt-Metz, D.; Black, D.S. Mindfulness-based interventions for obesity-related eating behaviours: A literature review. Obes. Rev. 2014, 15, 453-461. [CrossRef] 
56. Rossy, L. The Mindfulness-Based Eating Solution: Proven Strategies to End Overeating, Satisfy Your Hunger, and Savor Your Life; New Harbinger Publications: Oakland, CA, USA, 2016.

57. Christensen, L.; Brooks, A. Changing Food Preference as a Function of Mood. J. Psychol. 2006, 140, 293-306. [CrossRef]

58. Sze, K.Y.P.; Lee, E.K.P.; Chan, R.H.W.; Kim, J.H. Prevalence of negative emotional eating and its associated psychosocial factors among urban Chinese undergraduates in Hong Kong: A cross-sectional study. BMC Public Health 2021, 21, 1-10. [CrossRef]

59. Camilleri, G.M.; Méjean, C.; Kesse-Guyot, E.; Andreeva, V.; Bellisle, F.; Hercberg, S.; Péneau, S. The Associations between Emotional Eating and Consumption of Energy-Dense Snack Foods Are Modified by Sex and Depressive Symptomatology. J. Nutr. 2014, 144, 1264-1273. [CrossRef]

60. Klump, K.L.; Keel, P.K.; Racine, S.E.; Burt, S.A.; Neale, M.; Sisk, C.L.; Boker, S.; Hu, J.Y. The interactive effects of estrogen and progesterone on changes in emotional eating across the menstrual cycle. J. Abnorm. Psychol. 2013, 122, 131-137. [CrossRef]

61. van Strien, T.; van der Zwaluw, C.S.; Engels, R.C. Emotional eating in adolescents: A gene (SLC6A4/5-HTT)-Depressive feelings interaction analysis. J. Psych. Res. 2010, 44, 1035-1042. [CrossRef] [PubMed]

62. Shriver, L.H.; Dollar, J.M.; Calkins, S.D.; Keane, S.P.; Shanahan, L.; Wideman, L. Emotional Eating in Adolescence: Effects of Emotion Regulation, Weight Status and Negative Body Image. Nutrients 2021, 13, 79. [CrossRef] [PubMed]

63. Galmiche, M.; Déchelotte, P.; Lambert, G.; Tavolacci, M.P. Prevalence of eating disorders over the 2000-2018 period: A systematic literature review. Am. J. Clin. Nutr. 2019, 109, 1402-1413. [CrossRef] [PubMed] 